\title{
A NEURAL NETWORK BASED ADAPTIVE ALGORITHM FOR MULTIMEDIA QUALITY FAIRNESS IN WLAN ENVIRONMENTS*
}

\author{
Chiapin Wang, Tsungnan Lin
}

\author{
Dept. of Electrical Engineering \\ National Taiwan University, Taipei, Taiwan \\ Email: jamesw@cht.com.tw; tsungnan@ntu.edu.tw
}

\begin{abstract}
This paper investigates multimedia quality fairness in wireless LAN environments where channel are error-prone due to mobility and fading. The experimental results show that using fixed MAC arguments for nodes in heterogeneous channel conditions leads to unequal throughput performance and that may incur the degradation of multimedia QoS. To overcome the unfairness problem for provisioning QoS, we propose a cross-layer adaptation scheme by on-line adapting the multidimensional MAC-layer backoff parameters depending on the application-layer QoS requirements and PHY-layer channel conditions. Our solution is based on an optimization approach which utilizes neural networks to learn the cross-layer function. Simulation results demonstrate that our adaptive scheme can tackle a variety of channel condition to provide fair throughput for nodes in heterogeneous channel conditions.
\end{abstract}

\section{INTRODUCTION}

With the popularity of IEEE 802.11 based WLANs (wireless local area networks), there are increasing demands of multimedia services such as streaming video, VOWLAN (voice over WLAN) and net meeting for mobile users. While most WLANs employ DCF (Distributed Coordination Function) [1], a contention-based part of IEEE 802.11 MAC for channel access arbitration, the issue of fairness arises since the performance of multimedia QoS is essentially affected by the proportion of transmission medium utilization. Many previous efforts have addressed the improvement of 802.11 fairness from various perspectives depending on the transmission scenarios [2] [3] [4]. Nagesh et al. [2] discuss the unbalanced bandwidth sharing between uplink/downlink transmissions in an infrastructure WLAN and propose to grant a prior channel access for the AP (access point) to improve throughput fairness. Jun and Hung [3] tackle the throughput unfairness due to hidden terminal problems in multi-hop ad-hoc networks and propose an extension MAC protocol to achieve better fairness. Pong and Moors [4] investigate on

\footnotetext{
* This work was supported in part by Yulong Corp. under grant 95-S-C01A and by Taiwan National Science Council under grant 94-2219-E-002-017 and 94-2213-E-002-066.
}

the behavior of 802.11e temporal and throughput fairness in a single-hop network to discover the optimal setting of TXOP (transmission opportunity) and maximum contention window for the desired fairness and throughput performance.

In most of these existing approaches, the fairness is considered and improved from PHY-layer or MAC-layer perspectives with the metrics of transmission time and station throughput respectively. However, while Multimedia applications require specific quality in terms of throughput, delay, and packet loss rates, the achievement of lower-layer fairness may not be accordance with the satisfaction of application-layer QoS. Thus the fairness provision strategy for multimedia services should involve application-layer QoS indicators such as equal multimedia quality and guaranteed minimum quality [5]. Furthermore, the wireless channel condition is a key factor to the fairness performance since it essentially influences the effectiveness of packet deliveries. The existing approaches [2] [3] which only take into account perfect or identical channel conditions may be no more efficient to provide expected performance since the transmission qualities are probably unequal due to mobility, fading, interference factors, and so on.

This paper investigates the multimedia quality fairness in a lossy-channel wireless LAN which employs DCF mechanisms. Since the performance of multimedia quality fairness is decided by the QoS requirements, the transmission characteristics and the applied system arguments, we are thus motivated to propose a cross-layer adaptive scheme which dynamically determines the multidimensional arguments based on the application-layer requirements as well as the real-time PHY-layer characteristics. For investigation in this paper, we explore the average throughput as the QoS metric and the backoff parameters as adapted multidimensional arguments, which consist of initial window size, increasing factor, and retry limit. We utilize neural networks to on-line learn the crosslayer correlations and utilize a cost reward function to quantify the performance of multimedia quality fairness. Then we exploit the learned functions to evaluate the gradient of the cost reward function with respect to each backoff parameter by means of a back-propagation manner. 
Therefore the multidimensional parameters can be jointly determined for optimizing the fairness performance in terms of minimizing the cost reward function.

We conduct the simulation scenarios to evaluate the effectiveness of our adaptive scheme. The performance is evaluated by a fairness index and the performed throughputs with a specific requirement level. The results demonstrate that under a variety of channel condition, our adaptive scheme can effectively provide fair throughput by adapting backoff parameters according to channel conditions and QoS requirements. The remainder of this paper is organized as follows. Section 2 formalizes the addressed optimization problem associated with multimedia quality fairness. Section 3 presents our adaptive algorithm for solving this problem. In Section 4, we construct simulation scenarios to evaluate the effectiveness of the proposed adaptive scheme. Section 5 draws our conclusions.

\section{THE OPTIMIZATION PROBLEM DESCRIPTION}

To investigate the optimization problem associated with multimedia quality fairness, we use the average throughput as the QoS metric and the backoff parameters as adapted multidimensional arguments. Consider that there are $\mathrm{K}$ nodes in the network and each node transmits one traffic flow. To node $\mathrm{i}$, the adopted initial window size, increasing factor, and retry limit is $\mathrm{CW}_{\mathrm{i}, \mathrm{min}}, \sigma_{\mathrm{i}}$, and $\mathrm{L}_{\mathrm{i}, \text { retry }}$; the achievable average throughput is $\mathrm{T}_{\mathrm{i}}$, and the throughput requirement for QoS is $T_{-} T_{H R}$. Since throughput of a given node is affected by the adopted backoff parameters itself as well as that of other contending nodes, the overall throughput is then a correlation function $f(\cdot)$ associated with the joint setting of backoff parameters. That is,

$$
\left(\mathrm{T}_{1}, \mathrm{~T}_{2}, \ldots . . \mathrm{T}_{\mathrm{K}}\right)=f\left(C W_{1, \text { min }}, \sigma_{1}, L_{1, \text { retry }} \ldots \ldots . . . . C W_{K, \text { min }}, \sigma_{K}, L_{K, \text { retry }}\right)
$$

The parameters can be chosen based on an optimization approach by minimizing a cost reward function. The cost reward function is designed such that the multimedia quality fairness is optimized while the overall achievable throughput is close to the specific QoS requirements. The cost-reward function, $\mathrm{C}_{\mathrm{QoS}}$ is:

$$
\mathrm{C}_{Q o S}=\sum_{\mathrm{i}=1}^{\mathrm{K}} \frac{\left(T_{i}-T_{-} T H R_{i}\right)^{2}}{T_{-} T H R_{i}}
$$

, where the denominator $\mathrm{T}_{-} \mathrm{THR}_{\mathrm{i}}$ is utilized to normalize the difference between the achievable throughput and the corresponding requirement. $\mathrm{C}_{\mathrm{QoS}}$ will be minimized while the provided channel utilization for each traffic flow is fairly in proportion to $\mathrm{T}_{-} \mathrm{THR}_{\mathrm{i}}$.

To minimize $\mathrm{C}_{\mathrm{QoS}}$, the parameters are iteratively updated based on the gradient of $\mathrm{C}_{\mathrm{QoS}}$ with respect to each backoff parameter. To calculate the gradient, the knowledge of $f(\cdot)$ is needed. However, $f(\cdot)$ strongly depends on the communication characteristics of the network such as the collision probability among nodes and the channel condition of each node. For example, when the channel conditions of some nodes deteriorate due to mobility, the channel utilization of themselves as well as of other contending nodes will change and finally the achievable throughput is influenced. Hence $f(\cdot)$ is a nonlinear, complicated, and time-variant function that is rather difficult to be depicted with analytical formulas. We are thus motivated to exploit neural networks to model the complicated function $f(\cdot)$. Then the learned $\hat{f}(\cdot)$ is utilized to evaluate the gradient of CQoS with respect to each backoff parameter by the technique of the back-propagation and chain-rule manner. The modeling and optimizing process of our adaptive scheme will be described in the following section.

\section{THE PROPOSED NEURAL NETWORK BASED ADAPTIVE ALGORITHM}

Now we propose an adaptive algorithm which can dynamically adjust MAC parameters for achieving multimedia quality fairness based on each node's communication characteristic and its own QoS requirement. We exploit multilayer perceptron (MLP) which is the most common representative of neural networks to model the correlation function between the adopted backoff parameters and the achievable throughput, $f(\cdot)$. The architecture of the exploited MLP consists of $3 \mathrm{~K}-\mathrm{x}-\mathrm{K}$ sensory units at the input layer, the hidden layer, and the output layer respectively to model the nonlinear function from the $3 \mathrm{~K}$ backoff parameters to the corresponding $\mathrm{K}$ throughput. The output of the $i^{\text {th }}$ neuron at the $l^{\text {th }}$ layer can be described as:

$$
\begin{aligned}
& \mathrm{u}_{\mathrm{i}}(l)=\sum_{\mathrm{j}=1}^{\mathrm{N}_{l-1}} \omega_{\mathrm{ij}}(l) \mathrm{a}_{\mathrm{j}}(l-1)+\theta_{\mathrm{i}}(l) \\
& \mathrm{a}_{\mathrm{i}}(l)=\mathrm{h}\left(\mathrm{u}_{\mathrm{i}}(l)\right) \quad 1 \leq \mathrm{i} \leq \mathrm{N}_{l} ; \quad l=1,2
\end{aligned}
$$

where $\mathrm{N}_{l}$ is the number of neuron at the $l^{\text {th }}$ layer; $\mathrm{u}_{\mathrm{i}}(l)$ and $\mathrm{a}_{\mathrm{i}}(l)$ are the activation and output values of the $i^{\text {th }}$ neuron at the $l^{\text {th }}$ layer. The input units are represented by $\mathrm{a}_{\mathrm{i}}(0)$ and the output units by $\mathrm{a}_{\mathrm{i}}(2) . \omega_{\mathrm{ij}}(l)$ refers to the weight connecting the output from the $j^{\text {th }}$ neuron at the $(l-1)^{\text {th }}$ layer to the input of the $i^{\text {th }}$ neuron at the $l^{\text {th }}$ layer. $\theta_{\mathrm{i}}(l)$ refers to the bias associated with the $i^{\text {th }}$ neuron at the $l^{\text {th }}$ layer. The used transferring function $h$ is sigmoid at hidden layer and is linear at output layer.

The nonlinear function can be modeled with MLP gradually by recursively adjusting $\omega_{\mathrm{ij}}(l)$ and $\theta_{\mathrm{i}}(l)$ to minimize MSE (mean squares error) between throughput $T_{i}$ and actual outputs $\mathrm{a}_{\mathrm{i}}(2)$. That is,

$$
E=\frac{1}{2} \sum_{m=1}^{M} \sum_{i=1}^{K}\left(T_{i}^{(m)}-a_{i}^{(m)}(2)\right)
$$

where $\mathrm{M}$ is the number of teacher patterns. The universal approximation theorem [6] shows that MLP can approximate the nonlinear function to an arbitrary degree of the accuracy.

Now we describe our adaptive algorithm on adaptively determining the backoff parameters for minimizing the cost reward function, $\mathrm{C}_{\mathrm{Q} o \mathrm{~s}}$. Here we denote backoff parameter $\mathrm{i}$ 
of the $n^{\text {th }}$ adaptation as $\beta_{\mathrm{i}}^{(\mathrm{n})}$. The gradient-learning formula to minimize $\mathrm{C}_{\mathrm{QoS}}$ with respect to $\beta_{\mathrm{i}}^{\text {(n) }}$ is

$$
\begin{aligned}
& \beta_{\mathrm{i}}^{(\mathrm{n}+1)}=\beta_{\mathrm{i}}^{(\mathrm{n})}+\Delta \beta_{\mathrm{i}}^{(\mathrm{n})} \\
& \Delta \beta_{\mathrm{i}}^{(\mathrm{n})}=-\mu \cdot \partial \mathrm{C}_{\mathrm{Qos}} / \partial \beta_{\mathrm{i}}^{(\mathrm{n})}, 1 \leq \mathrm{i} \leq 3 K
\end{aligned}
$$

where $\mu$ is the adjusting rate. To evaluate $-\partial C_{\mathrm{Qos}} / \partial \beta_{\mathrm{i}}^{(\mathrm{n})}$, a chain-rule technique is utilized:

$$
-\partial \mathrm{C}_{\mathrm{Qos}} / \partial \beta_{\mathrm{i}}^{(\mathrm{n})}=\lambda_{\mathrm{i}}^{(\mathrm{n})}(1) \cdot\left(\partial \mathrm{a}_{\mathrm{i}}{ }^{(\mathrm{n})}(1) / \partial \beta_{\mathrm{i}}^{(\mathrm{n})}\right)
$$

where $\lambda_{1}^{(\mathrm{n})}(l)$ is defined as the minus gradient of $\mathrm{C}_{\mathrm{Qos}}$ with $\mathrm{a}_{\mathrm{i}}^{(\mathrm{n})}(l): \lambda_{\mathrm{i}}^{(\mathrm{n})}(l) \equiv-\partial \mathrm{C}_{\mathrm{Qos}} / \partial \mathrm{a}_{\mathrm{i}}{ }^{(\mathrm{n})}(l) \cdot \lambda_{\mathrm{i}}^{(\mathrm{n})}(l)$ can be derived with $\lambda_{i}^{(n)}(l+1)$ from upper layer with a chain-rule manner:

$$
\lambda_{\mathrm{i}}^{(\mathrm{n})}(l)=\sum_{\mathrm{j}=1}^{\mathrm{N}_{t+1}} \lambda_{\mathrm{j}}^{(\mathrm{n})}(l+1) h^{\prime}\left(u_{j}(l+1)\right) w_{j i}(l+1)
$$

In particular, the minus gradient of $\mathrm{C}_{\mathrm{Q} o \mathrm{~S}}$ with the output layer, $\lambda_{i}^{(n)}(2)$ can be derived from the cost reward function as shown in equation (2). By back-propagation and chainrule manners, $\lambda_{i}^{(n)}(l)$ can be derived in the order of $l=2,1$ and 0 . Since the value of $\mathrm{a}_{\mathrm{i}}^{(\mathrm{n})}(0)$ is equal to $\beta_{i}^{(\mathrm{n})}$, equation (6) becomes

$$
\beta_{\mathrm{i}}^{(\mathrm{n}+1)}=\beta_{\mathrm{i}}^{(\mathrm{n})}+\mu \lambda_{\mathrm{i}}^{(\mathrm{n})}(0)
$$

From equation (10), the set of multidimensional backoff parameters for minimizing $\mathrm{C}_{\mathrm{QoS}}$ is determined.

The proposed adaptive algorithm recursively and alternately models the function and determines backoff parameters for minimizing $\mathrm{C}_{\mathrm{Qos}}$. To summarize, the block diagram of this algorithm is shown in Fig. 1 which consists of the following 4 steps:

Step1: Collecting up-to-date training data. M most recent data of the adopted backoff parameters and the corresponding throughputs are collected for the teacher patterns.

Step2: On-line modeling the nonlinear function. With up-todate teacher patterns, present $f(\cdot)$ is modeled as $\hat{f}(\cdot)$ gradually by recursively adjusting the weights and biases of MLP till the MSE between the teacher values and actual MLP outputs falls below a prescribed level.

Step3: Determining backoff parameters. The learned $\hat{f}(\cdot)$ is utilized to evaluate the gradient of $\mathrm{C}_{\mathrm{QoS}}$ with respect to each backoff parameter by the technique of back-propagation manners. With these gradients the set of multidimensional backoff parameters for minimizing $\mathrm{C}_{\mathrm{Qos}}$ can then be computed.

Step4: Applying new backoff parameters and refreshing training data. New parameters are adopted into the system. Therefore $\mathrm{M}$ most recent data are used for the teacher patterns. The process returns back to Step 1 to keep learning $f(\cdot)$ in accordance with current wireless circumstances.

To evaluate the performance of our proposed algorithm, we use the Jain fairness index [4]. It is a pertinent criterion index since our investigation focuses on the scenario that the participating nodes are of the same class with similar QoS requirements. This index is represented as:

$$
\text { Jain fairness index }=\frac{\left(\sum_{i=1}^{\mathrm{K}} x_{i}\right)^{2}}{\mathrm{~K} \sum_{i=1}^{\mathrm{K}} x_{i}{ }^{2}}
$$

where $\mathrm{K}$ is the number of contending nodes of identical class and $x_{i}$ refers to achievable throughput by node i. The index has a range of $(0,1]$ to evaluate the fairness.

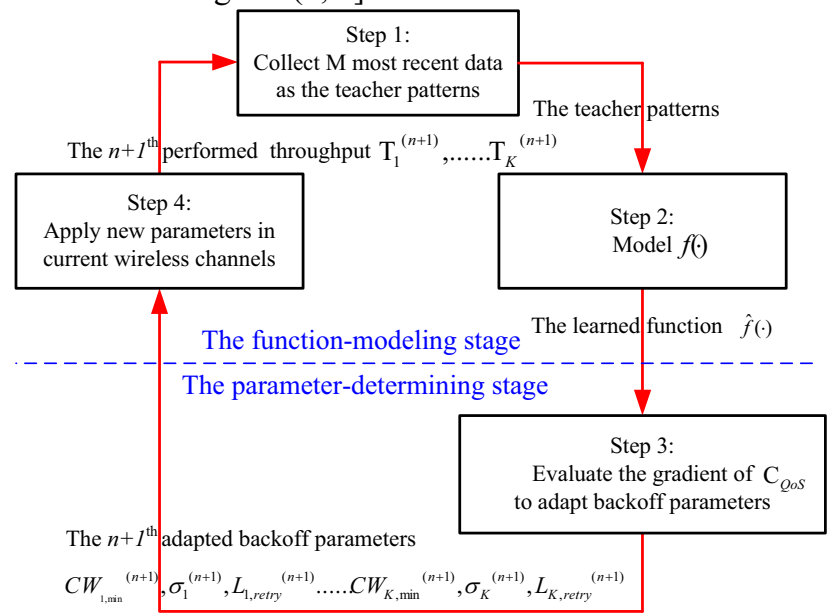

Fig. 1 The Block diagram of the proposed adaptive algorithm

\section{EXPERIMENTS SETUP AND RESULTS}

To demonstrate the effectiveness of our proposed algorithm, the simulation environment is set up as follows. There are 4 nodes in an infrastructure IEEE 802.11 WLAN. Each node transmits a saturated traffic flow (i.e. every node always has a packet to transmit) to the AP with basic CSMA/CA scheme. All the nodes are of the same class and with an identical throughput requirement of $160 \mathrm{Kbps}$ for basic QoS. Assume that half the nodes ( 2 nodes), named ideal-channel (IC) nodes, are always in an ideal channel; the others, named error-prone-channel (EC) nodes, are initially with an average BER of $2 \mathrm{E}-5$ and later suffer from quality degradation with an average BER of 4E-5 when they move away from AP.

We compare the proposed adaptive scheme with IEEE 802.11 DCF protocol which adopts fixed backoff parameters: the initial window size of 32, increasing factor of 2 , and retry limit of 5 [1]. For our adaptive algorithm, the adaptation space of initial window size is from 8 to 64 , increasing factor from 1.1 to 4 , and retry limit from 1 to 10 . The chosen values of initial window size and retry limit will be rounded to the closest integer. The backoff parameters and throughputs performed in the last 5 adaptation sequences are utilized as the teacher patterns. In the function-modeling stage, the weights and biases of MLP will be recursively updated till the MSE falls below $1 \mathrm{E}-6$ or the training epochs are over 1000 times. We write Matlab codes to evaluate the saturated throughputs of IEEE 802.11 WLANs with various values of backoff parameter values in error-prone channel conditions. The system parameters adopted are shown in Table 1. 


\begin{tabular}{|c|c|c|c|}
\hline $\begin{array}{c}\text { Payload } \\
=1023 \text { bytes }\end{array}$ & $\begin{array}{c}\text { MAC header } \\
=34 \text { bytes }\end{array}$ & $\begin{array}{c}\text { DIFS } \\
=50 \mathrm{us}\end{array}$ & $\begin{array}{c}\text { Initial window size } \\
=32\end{array}$ \\
\hline $\begin{array}{c}\text { Slot time } \\
=20 \mathrm{us}\end{array}$ & $\begin{array}{c}\text { PHY header } \\
=16 \text { bytes }\end{array}$ & $\begin{array}{c}\text { SIFS } \\
=10 \mathrm{us}\end{array}$ & $\begin{array}{c}\text { Increasing factor } \\
=2\end{array}$ \\
\hline $\begin{array}{c}\text { Data rate } \\
=1 \mathrm{Mbps}\end{array}$ & $\begin{array}{c}\text { ACK } \\
=64 \text { bytes }\end{array}$ & $\begin{array}{c}\text { Propagation delay } \\
=1 \mathrm{us}\end{array}$ & $\begin{array}{c}\text { Retry limit } \\
=5\end{array}$ \\
\hline
\end{tabular}

Table 1 System parameters

Fig. 2 shows the fairness index $F_{\text {Qos }}$ with $802.11 \mathrm{DCF}$ and the proposed adaptive scheme respectively. It is shown that $\mathrm{F}_{\mathrm{Qos}}$ with $802.11 \mathrm{DCF}$ is initially as $94.9 \%$ and drops to $82.7 \%$ as the difference of channel condition within nodes become larger during the iteration of 11 (average BER of EC nodes becomes from $2 \mathrm{E}-5$ to $4 \mathrm{E}-5$ ). With our adaptive scheme, the backoff parameters are changed adaptively. It is shown in Fig. 2 that $F_{Q o s}$ with the adaptive scheme increases and converges to 1 after the $5^{\text {th }}$ sequence. Later $\mathrm{F}_{\mathrm{Qos}}$ drops at the iteration of 11 because channel conditions change at this moment. It is shown that $\mathrm{F}_{\mathrm{QoS}}$ converges to 1 again after the $16^{\text {th }}$ sequence. The results demonstrate that our adaptive algorithm can effectively adjust the multidimensional parameters under heterogeneous channel conditions to improve fairness in terms of maximizing $\mathrm{F}_{\mathrm{Qos}}$.

Fig. 3 presents the saturated throughput with 802.11 DCF and the proposed adaptive scheme respectively. It is shown that throughput of an EC node with 802.11 DCF (denoted by the circle) is $151.7 \mathrm{Kbps}$ initially and comes to $104 \mathrm{Kbps}$ as channel conditions changes. With 802.11 DCF, EC hosts cannot meet the requirement for minimum QoS $(160 \mathrm{Kbps})$. In the meanwhile throughput of an IC node is $243.5 \mathrm{Kbps}$ initially and comes to $279.5 \mathrm{Kbps}$ (denoted by the triangle), exceeding the prescribed requirement a lot. It is demonstrated that fairness can not be achieved by the approach of fixed parameters in a varying heterogeneous wireless environment.

With the proposed adaptive scheme, it is shown that the throughput fairness is significantly improved. Throughput of IC and EC nodes are almost equal (194 Kbps) after the $5^{\text {th }}$ iteration which can meet QoS requirements well. It is the reason for that $\mathrm{F}_{\mathrm{Qos}}$ converges to 1 at the $5^{\text {th }}$ sequence (referring to Fig. 2). While channel conditions change, the adapted parameters cease to be effective in the present situation. Consequently the throughput of an EC host degrades to $131.5 \mathrm{Kbps}$ and can no more satisfy the QoS. With the proposed algorithm on-line learning the cross-layer current function and adjusting parameters, it is shown that the throughputs of an IC and EC host are almost equal $(177 \mathrm{Kbps})$ after the $16^{\text {th }}$ sequence which can again meet the requirement QoS. The results demonstrate that our adaptive algorithm can on-line determine the multidimensional MAC parameters to improve multimedia quality fairness under a variety of wireless circumstances.

\section{CONCLUSION}

This paper investigates on the multimedia quality fairness in a lossy-channel wireless LAN which uses DCF mechanisms. We propose a cross-layer adaptive algorithm which tackles the time-varying channels to perform fairness by real-time determining the multidimensional MAC-layer backoff parameters based on each node's PHY-layer communication characteristics and its own application-layer QoS requirement. The simulation results demonstrate that our adaptive scheme can effectively guaranty fair QoS under varying heterogeneous channel conditions.

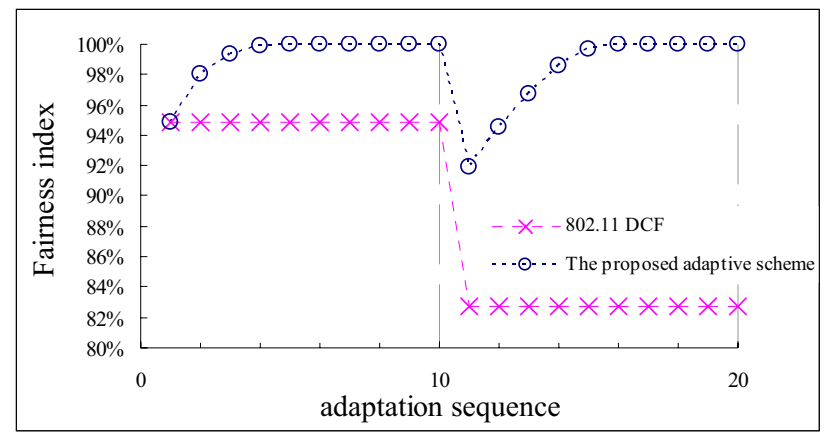

Fig. 2 The fairness index with fixed and adaptive parameters

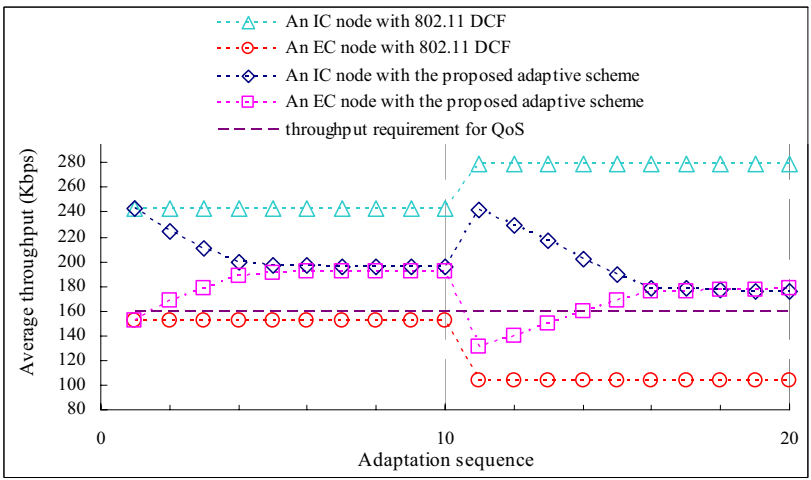

Fig. 3 The saturated throughput with fixed and adaptive parameters

\section{REFERENCES}

[1] IEEE 802.11 WG, Part 11: Wireless LAN Medium Access Control (MAC) and Physical Layer (PHY) specification, 1999

[2] Nagesh Nandiraju, H. Gossain, D. Cavalcanti, K. Chowdhury, D. P. Agrawal, Achieving Fairness in Wireless LANs by Enhanced 802.11 DCF. U.C. ECECS Technical Report August 2005

[3] Jun He and Hung Keng Pung, A fairer multiple access protocol for multi-hop wireless networks: hybrid asynchronous time division multiple access protocol (HATDMA) Local Computer Networks, 2003. Proceedings. 28th Annual IEEE International Conference on 20-24 Oct. 2003 Page(s):356 - 365

[4] Dennis Pong and Tim Moors, Fairness and capacity trade-off in IEEE 802.11 WLANs. Local Computer Networks, 2004. 29th Annual IEEE International Conference on 16-18 Nov. 2004 Page(s):310-317

[5] Mihaela van Der Schaar, Davis Sai Shankar N, Cross-layer wireless multimedia transmission: challenges, principles, and new paradigms, Wireless Communications, IEEE Volume 12, Issue 4, Aug. 2005 Page(s):50 - 58

[6] K. Hornik, M. Stinchcombe, H. White, Multilayer feedforward networks are universal approximators, Neural Networks Volume 2, Issue 51989 Pages: $359-366$ 